\title{
One-Step Preparation of Poly-Lactic-Co-Glycolic-Acid Microparticles to Prevent the Initial Burst Release of Encapsulated Water-Soluble Proteins
}

\author{
Hiroyuki Takabe ${ }^{1,2}$, Moriyuki Ohkuma ${ }^{1}$, Yasunori Iwao ${ }^{2}$, Shuji Noguchi ${ }^{2}$, Shigeru Itai $^{2 *}$ \\ ${ }^{1}$ Drug Formulation Department, Central Research Laboratories, Kaken Pharmaceutical Co., Ltd. Shizuoka, Japan; ${ }^{2}$ Department of \\ Pharmaceutical Engineering, Graduate School of Pharmaceutical Sciences, University of Shizuoka, Shizuoka, Japan. \\ Email: ${ }^{*}$ s-itai@u-shizuoka-ken.ac.jp
}

Received September $18^{\text {th }}, 2013$; revised October $12^{\text {th }}, 2013$; accepted October $22^{\text {nd }}, 2013$

Copyright (C) 2013 Hiroyuki Takabe et al. This is an open access article distributed under the Creative Commons Attribution License, which permits unrestricted use, distribution, and reproduction in any medium, provided the original work is properly cited.

\begin{abstract}
An initial burst is often observed during the release of active pharmaceutical ingredients (APIs) from poly-lactic-coglycolic-acid (PLGA) microparticles (MPs) which have been prepared by the emulsion-solvent evaporation method. Herein, we describe the development of a simple one-step coating method that suppresses the initial burst release process. This new method involves coating the PLGA-MPs with PLGA, with the coating process being performed through the phase separation of PLGA on the surface of PLGA-MPs using the emulsion-solvent evaporation method. Bovine serum albumin (BSA) was encapsulated in the PLGA-MPs as a model API. The coated MPs were spherical in shape with no pores on their smooth surface, whereas the non-coated PLGA-MPs had porous surfaces. An in vitro release study showed that the residual levels of BSA in the coated and non-coated PLGA-MPs after $1 \mathrm{~h}$ were about $99 \%$ and $16 \%$ of the original loads, respectively. The one-step coating method therefore represents a useful method for preparing PLGA-MPs that do not give an initial burst release of proteinaceous APIs.
\end{abstract}

Keywords: Poly-Lactic-Co-Glycolic-Acid; Microparticle; Suppression of Initial Burst Release; Coating; Bovine Serum Albumin

\section{Introduction}

Biocompatible and biodegradable synthetic polymers such as poly-lactic-co-glycolic-acid (PLGA) have been used as base materials in a number of different functional materials, such as absorbable biomaterial implants [1,2], absorbable surgical sutures $[3,4]$ and microparticles (MPs) for the sustained release of active pharmaceutical ingredients (APIs) including bioactive proteins [5]. When highly water-soluble proteins are encapsulated in MPs, an initial burst release of the proteins has been often observed [6,7]. The development of methods capable of suppressing these initial burst releases is necessary to enhance the therapeutic effects of APIs and reduce their side effects. To date, several methods have been developed to suppress the initial burst release of APIs from PLGA-MPs, including the replacement of PLGA with different materials with slower degradation rates [8-10],

"Corresponding author. and the coating of the MPs with cationic compounds [11-14] or water-soluble polymers [11-15]. Ahmed et al. [16] recently reported a new method for coating PLGAMPs with PLGA. According to this method, however, the PLGA-MPs had to be dispersed in peanut oil to enable the formation of an oil layer on their surface to prevent the dissolution and aggregation of PLGA-MPs during the PLGA coating, indicating that this coating method still required a series of time-consuming steps. In addition, the application of this method could lead to significant difficulties in terms of the associated cleaning processes, because of oil in oil mixing, and concerns therefore remain that the final products could be contaminated with oil. One of the major advantages of PLGA coatings is that the encapsulated APIs can be released from the PLGA-MPs at a constant rate because the degradation rates of PLGA are similar in the MPs and the coating layer. Taken together, there is an urgent need in the field of pharmaceutical research for a simple and effective 
method of coating PLGA-MPs with PLGA [17].

In the current study, we have developed a simple onestep method for coating PLGA-MPs with PLGA during the final step of the emulsion-solvent evaporation method. With regards to the emulsion-solvent evaporation method, PLGA phase separation occurred at the interface between the PLGA and aqueous solutions during the preparation of the PLGA-MPs [6,8-10]. Following the preparation of the PLGA-MPs, the addition of a PLGA solution effectively induced PLGA phase separation at the surface of the PLGA-MPs, and the MPs were consequently coated with PLGA. PLGA-MPs encapsulated with bovine serum albumin (BSA) (BSA-MPs) were coated with PLGA using the current method to evaluate the general utility of this one-step coating method as a means of suppressing the initial burst release of proteinaceous APIs (Figure 1), and their physicochemical properties and protein-release behaviors were also investigated.

\section{Experimental}

\subsection{Materials}

BSA (ELISA applications) and sodium dodecyl sulfate (SDS) were purchased from Sigma-Aldrich (St. Louis, MO, USA). PLGA-7510 and PLGA-7520 were purchased from Wako Pure Chemical Industries Ltd. (Osaka, Japan). Phosphate-buffered saline (PBS) tablets were purchased from Takara Bio Inc. (Shiga, Japan). All of the reagents used in the current study were purchased in the highest grade commercially available.

\subsection{Preparation of BSA-MPs}

PLGA-7510 (1.0 g) was dissolved in dichloromethane (DCM, $1.5 \mathrm{~mL}$ ), and $0.25 \mathrm{~mL}$ of $16.0 \mathrm{mg} / \mathrm{mL}$ BSA aqueous solution was added to the resulting solution and homogenized at $16,000 \times \mathrm{g}$ for 30 seconds to obtain a water-in-oil (W/O) emulsion. The W/O emulsion was added to $150 \mathrm{~mL}$ of $0.1 \%$ PVA aqueous solution in a drop-wise manner and homogenized at $7000 \mathrm{rpm}$ for 60 seconds to prepare a water-in-oil-in-water $(\mathrm{W} / \mathrm{O} / \mathrm{W})$ emulsion. The DCM was then removed from the emulsion using the emulsion-solvent evaporation method under stirring for $3 \mathrm{~h}$ at ambient temperature. The resulting BSA-MPs were collected by centrifugation at $1600 \times \mathrm{g}$ for $15 \mathrm{~min}$, with the supernatant being decanted. Distilled water was then added to the MPs to wash out any free BSA and PVA, with the resulting material being separated by centrifugation. This washing process was repeated five times, and the resulting washed BSA-MPs were collected and lyophilized for $48 \mathrm{~h}$.

\subsection{Preparation of BSA-MPs 7510 Coating- 1 and BSA-MPs 7520 Coating-1}

The PLGA-7510 and PLGA-7520 coating solutions were prepared by dissolving $100 \mathrm{mg}$ of PLGA-7510 or PLGA7520 in $2.0 \mathrm{~mL}$ of acetone. The resulting PLGA-coating solutions were added to the $\mathrm{W} / \mathrm{O} / \mathrm{W}$ emulsion prepared as described above with stirring. These emulsions were stirred for $3 \mathrm{~h}$ at ambient temperature to allow for the removal of acetone by evaporation. The PLGA-coated MPs (BSA-MPs 7510 coating-1 and BSA-MPs 7520 coating-1, respectively) were then washed and lyophilized as in the BSA-MP preparation.

\subsection{Preparation of BSA-MPs 7510 Coating-2}

A portion of the lyophilized BSA-MPs as prepared in Section $2.2(1.0 \mathrm{~g})$ was dispersed in $150 \mathrm{~mL}$ of a $0.1 \%$ PVA aqueous solution, and the resulting dispersion was added to $2.0 \mathrm{~mL}$ of a PLGA-7510 coating solution. The mixture was then stirred for $3 \mathrm{~h}$ at $40^{\circ} \mathrm{C}$. The MPs coated with PLGA-7510 (BSA-MPs 7510 coating-2) were



Figure 1. Schematic representation of the PLGA coating process using the one-step emulsion-solvent evaporation method. 
washed and lyophilized as in the BSA-MP preparation.

\subsection{Particle Size Distribution}

The particle size distributions of the different PLGAMPs were determined in a wet process using a laser scattering particle size analyzer (LDSA-1500A, Tonichi Computer Applications Co., Ltd., Tokyo, Japan).

\subsection{Scanning Electron Microscopy (SEM)}

The surface morphologies of the different PLGA-MPs were assessed by SEM (JSM-6390LA, JEOL Ltd., Tokyo, Japan). The samples were sputter-coated with platinum under vacuum prior to imaging.

\subsection{Determination of the BSA Content in MPs}

Four milligram samples of the MPs were added to a 4:1 $(\mathrm{v} / \mathrm{v})$ mixture of acetone and water $(1 \mathrm{~mL})$, and the resulting solutions were agitated for $1 \mathrm{~h}$ at ambient temperature. These solutions were centrifuged for $15 \mathrm{~min}-$ utes at $16,000 \times \mathrm{g}$ to remove supernatants. The pellets thus obtained were washed with $1.6 \mathrm{~mL}$ of acetone and dried at ambient temperature for 30 minutes. The dried pellets were dissolved in $0.1 \mathrm{~mL}$ of a $0.5 \%$ SDS solution by sonication (US-105, SND Co., Ltd., Nagano, Japan) over a 15 minute period. The concentrations of BSA in the solutions were determined using a protein quantification kit CBQCA (Molecular Probes, Eugene, Oregon). The BSA contents of the PLGA-MPs, as well as the actual drug loading and encapsulation efficiency values of the materials were calculated according to the following equations.

$$
\begin{aligned}
& \text { Actual drug loading }(\%) \\
& =\frac{\text { BSA in PLGA MPs }(\mathrm{mg})}{\operatorname{MPs}(\mathrm{mg})} \times 100 \\
& \text { Encapsulation efficiency }(\%) \\
& =\frac{\text { Actual drug loading }(\%)}{\text { Theoretical drug loading }(\%)} \times 100
\end{aligned}
$$

\subsection{Release Studies}

Four milligram samples of the different MPs were dispersed in $0.4 \mathrm{~mL}$ of a PBS buffer in a micro-tube and incubated at $37^{\circ} \mathrm{C}$. The micro-tubes were subsequently collected at pre-determined time points and centrifuged for 15 minutes at $1600 \times \mathrm{g}$ to remove the supernatants. The MPs were then washed with $1 \mathrm{~mL}$ of distilled water, and the levels of residual BSA in the MPs were quantified using CBQCA as described above. The ratio of residual BSA in the MPs was calculated according to the following equation.

\section{Results and Discussion}

BSA-MPs, which were prepared using the emulsionsolvent evaporation method, were coated in a one-step operation to suppress the initial burst process. More specifically, phase separation occurred at the surface of the MPs when PLGA solution was added to a dispersion of MPs in a poor solvent, and the MPs were consequently coated with PLGA. Using this one-step coating methods, three kinds of PLGA-coated BSA-MPs were prepared: 1) BSA-MPs coated with PLGA-7510 with a lactide: glycolide monomer composition of 75:25, and an average molecular weight of 10,000 that were subsequently named BSA-MPs 7510 coating-1; 2) BSA-MPs coated with PLGA-7520 with an average molecular weight of 20,000 that were subsequently named BSA-MPs 7520 coating-1; and 3) BSA-MPs that were coated with PLGA 7510 following a lyophilization process that were subsequently named BSA-MPs 7510 coating-2. The yield of the BSA-MPs was approximately $75 \%$, whereas those of the coated BSA-MPs were in the range of $80 \%-85 \%$ (Table 1). The higher yields observed for the coated MPs were attributed to the amount of PLGA coating. The yield of BSA-MPs 7510 coating-2 was lower than that of BSA-MPs 7510 coating-1, likely because of the incremental lyophilization process.

The median diameter $\left(X_{50}\right)$ values of the BSA-MPs and the BSA-MPs 7510 coating-2 particles were 24.4 and $23.2 \mu \mathrm{m}$, respectively, whereas the $X_{50}$ of the BSA-MPs 7510 coating-1 particles was higher at $31.8 \mu \mathrm{m}$ (Figure 2, Table 1). A mean diameter in the range of 20 to $30 \mu \mathrm{m}$ is generally required for the MPs to possess good syringeability. Pleasingly, the $X_{50}$ values of the MPs involved in the current study fell within or were just outside of this range. The $X_{50}$ of the BSA-MPs 7520 coating-1 particles, however, was $39.1 \mu \mathrm{m}$, and therefore out of the preferred range. The higher viscosity of PLGA 7520 [18-20] may have enhanced its ability to adhere to the MP surfaces during the coating step, and resulted in its large $X_{50}$.

Although many pores of about $2 \mu \mathrm{m}$ in diameter were observed on the surfaces of the BSA-MPs, no pores were found on the smooth surfaces of the spherical BSA-MPs coated with PLGA (Figure 3). These results indicated that the pores on the surfaces of the MPs had been efficiently covered with PLGA as a consequence of this onestep coating procedure.

The encapsulation efficiencies of the PLGA-coated MPs were all lower than that of the BSA-MPs (Table 1), likely because of the leakage of BSA during the coating process. The encapsulation efficiencies of the three coated MPs prepared during the course of the current study were particularly low, especially for BSA-MPs 
Table 1. Yields and particle properties of the BSA-MPs and BSA-MPs coated with PLGA.

\begin{tabular}{|c|c|c|c|c|c|c|}
\hline \multirow{2}{*}{ MPs } & \multirow{2}{*}{ Yield $^{*}(\%)$} & \multicolumn{3}{|c|}{ Particle size distribution $(\mu \mathrm{m})$} & \multirow{2}{*}{ BSA loading (\%) } & \multirow{2}{*}{ Encapsulation efficiency (\%) } \\
\hline & & $X_{10}$ & $X_{50}$ & $X_{90}$ & & \\
\hline BSA-MPs & 76.2 & $9.6 \pm 1.1$ & $24.4 \pm 0.9$ & $50.5 \pm 0.6$ & $0.25 \pm 0.02$ & $65.5 \pm 5.4$ \\
\hline BSA-MPs 7510 coating-1 & 83.6 & $12.0 \pm 0.6$ & $31.8 \pm 0.2$ & $52.8 \pm 0.1$ & $0.23 \pm 0.02$ & $57.3 \pm 4.4$ \\
\hline BSA-MPs 7520 coating- 1 & 81.1 & $16.6 \pm 0.2$ & $39.1 \pm 0.1$ & $63.9 \pm 0.1$ & $0.18 \pm 0.02$ & $44.2 \pm 4.0$ \\
\hline BSA-MPs 7510 coating- 2 & 81.8 & $10.2 \pm 0.4$ & $23.2 \pm 0.1$ & $55.1 \pm 1.1$ & $0.16 \pm 0.01$ & $41.0 \pm 1.9$ \\
\hline
\end{tabular}

* Yield of the PLGA-MPs $(\%)=\frac{\text { Recovered amount of PLGA-MPs }(\mathrm{g})}{\text { input PLGA }(\mathrm{g})} \times 100$.
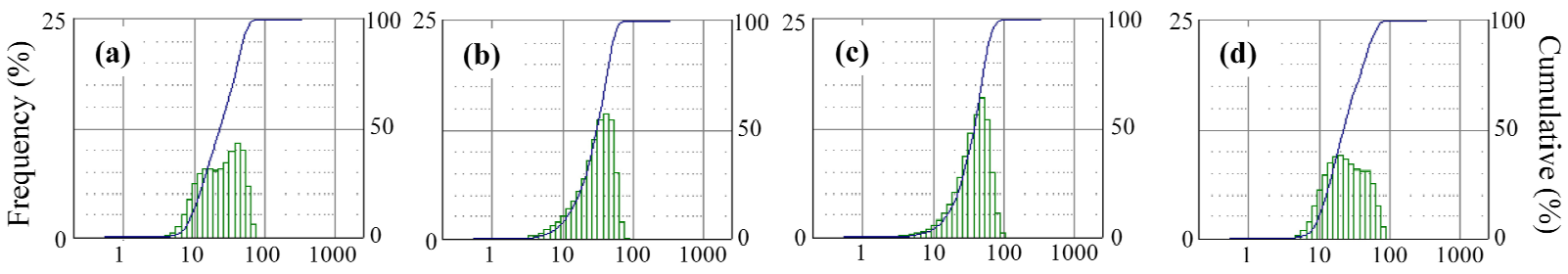

Particles size $(\mu \mathrm{m})$

Figure 2. Particle size distribution of the MPs. (a) BSA-MPs; (b) BSA-MPs 7510 coating-1; (c) BSA-MPs 7520 coating-1; and (d) BSA-MPs 7510 coating-2. The bar and line charts show the frequency (\%) and cumulative values (\%), respectively.
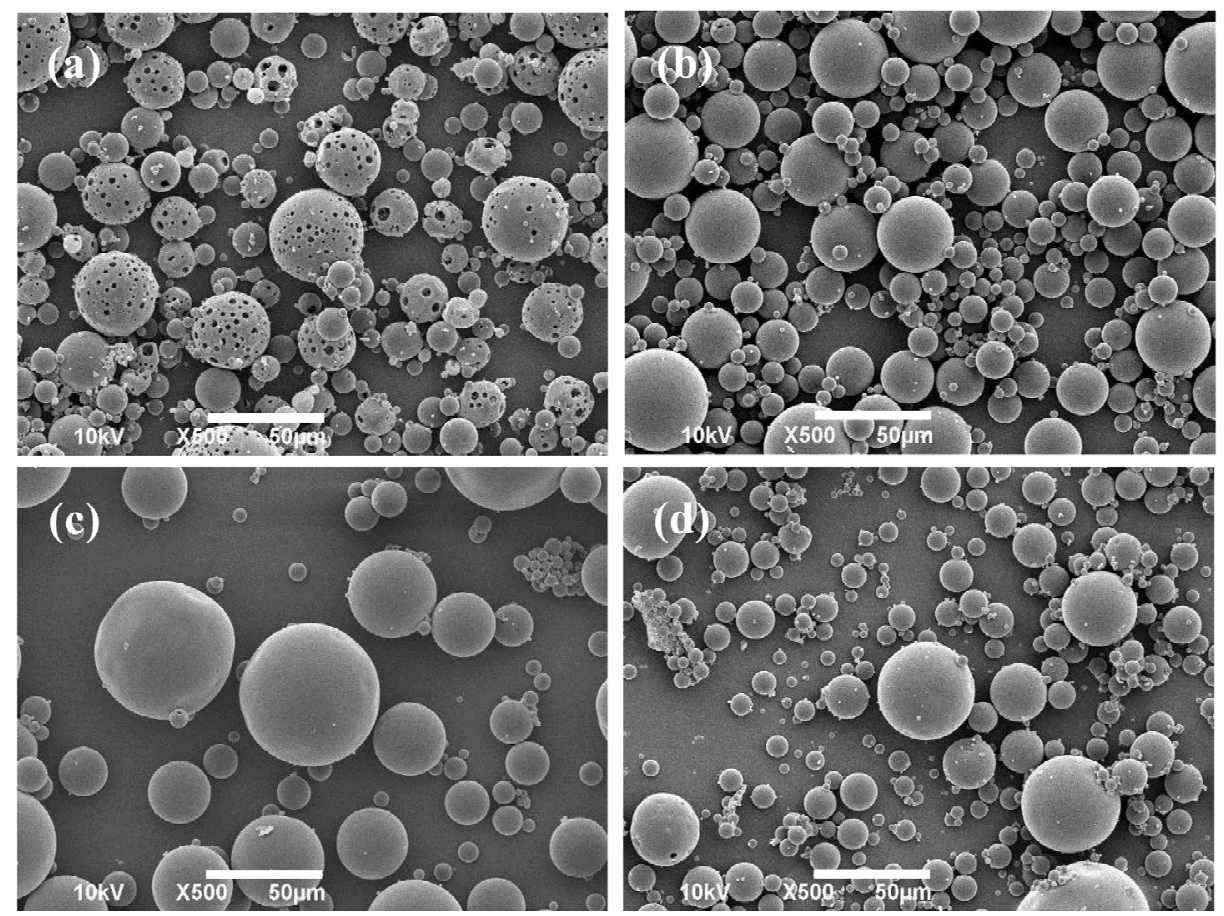

Figure 3. SEM images of MPs. (a) BSA-MPs; (b) BSA-MPs 7510 coating-1; (c) BSA-MPs 7520 coating-1; and (d) BSA-MPs 7510 coating- 2 .

7510 coating- 2 and BSA-MPs 7520 coating- 1 . For the preparation of BSA-MPs 7510 coating-2, an incremental process including a lyophilization step was needed. For the preparation of BSA-MPs 7520 coating-1, the PLGA 7520 in the coating solution might not solidify rapidly on the core surface because of its high viscosity [18-20], and this resulted in more BSA leaking from the MPs and the observed low encapsulation efficiency.

The BSA-MPs showed an initial burst release with $84 \%$ of the BSA being released from the MPs following $1 \mathrm{~h}$ of the release study (Figure 4(a)). The initial burst release process could be induced by the rapid penetration 

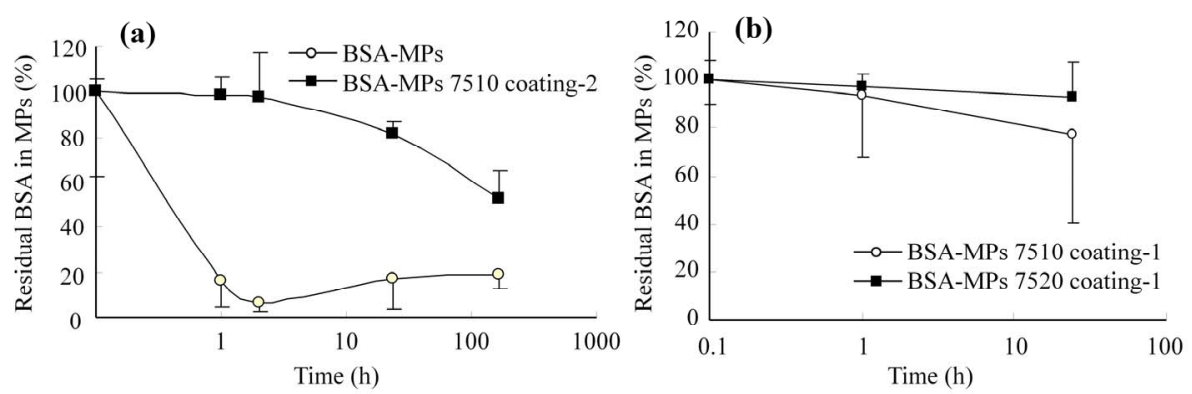

Figure 4. BSA release studies. (a) MPs with and without the PLGA coating; and (b) MPs coated with two types of PLGA. Each point represents the average value from three measurements $( \pm \mathrm{SD})$.

of the external solvent into the BSA-MPs through the many pores observed on their surfaces. In contrast, BSAMPs 7510 coating-2 showed no initial burst release, with $99 \%$ of the BSA remaining in the MPs following $1 \mathrm{~h}$ of the release study. This difference can be explained by the blockage of the pores on the surfaces of the MPs by the PLGA coating. Thereafter, the BSA-MPs 7510 coating-2 released $40 \%$ of the encapsulated BSA in sustained manner over $168 \mathrm{~h}$. The release rate of the BSA was found to be dependent on the type of PLGA used for the coating. About $80 \%$ of the BSA remained in BSA-MPs 7510 coating- 1 following $24 \mathrm{~h}$ of the release study, whereas more than $90 \%$ of the BSA remained in BSAMPs 7520 coating-1 following the same time period (Figure 4(b)). These results suggest that it would be possible to design PLGA-MPs with different release rates by optimizing the average molecular weight of the PLGA used for the coating process.

\section{REFERENCES}

[1] R. A. Jain, "The Manufacturing Techniques of Various Drug Loaded Biodegradable Poly(Lactide-Co-Glycolide) (PLGA) Devices," Biomaterials, Vol. 21, No. 23, 2000, pp. 2475-2490.

http://dx.doi.org/10.1016/S0142-9612(00)00115-0

[2] P. I. P. Park, M. Makoid and S. Jonnalagadda, "The Design of Flexible Ciprofloxacin-Loaded PLGA Implants Using a Reversed Phase Separation/Coacervation Method," European Journal of Pharmaceutics and Biopharmaceutics, Vol. 77, No. 2, 2011, pp. 233-239. http://dx.doi.org/10.1016/j.ejpb.2010.11.014

[3] K. Andreas, R. Zehbe, M. Kazubek, K. Grzeschik, N. Sternberg, H. Bäumler, H. Schubert, M. Sittinger and J. Ringe, "Biodegradable Insulin-Loaded PLGA Microspheres Fabricated by Three Different Emulsification Techniques: Investigation for Cartilage Tissue Engineering," Acta Biomaterialia, Vol. 7, No. 4, 2011, pp. 1485-1495. http://dx.doi.org/10.1016/j.actbio.2010.12.014

[4] C. B. Weldon, J. H. Tsui, S. A. Shankarappa, V. T. Nguyen, M. Ma, D. G. Anderson and D. S. Kohane, "Electrospun Drug-Eluting Sutures for Local Anesthesia," Journal of Controlled Release, Vol. 161, No. 3, 2012, pp. 903-
909. http://dx.doi.org/10.1016/j.jconrel.2012.05.021

[5] T. Niwa, H. Takeuchi, T. Hino, M. Nohara and Y. Kawashima, "Biodegradable Submicron Carriers for Peptide Drugs: Preparation of dl-Lactide/Glycolide Copolymer (PLGA) Nanospheres with Nafarelin Acetate by a Novel Emulsion-Phase Separation Method in an Oil System," International Journal of Pharmaceutics, Vol. 121, No. 1, 1995 , pp. $45-54$.

http://dx.doi.org/10.1016/0378-5173(95)00002-Z

[6] S. Mao, J. Xu, C. Cai, O. Germershaus, A. Schaper and T. Kissel, "Effect of WOW Process Parameters on Morphology and Burst Release of FITC-Dextran Loaded PLGA Microspheres," International Journal of Pharmaceutics, Vol. 334, No. 1-2, 2007, pp. 137-148. http://dx.doi.org/10.1016/j.ijpharm.2006.10.036

[7] Y. Yamaguchi, M. Takenaga, A. Kitagawa, Y. Ogawa, Y. Mizushima and R. Igarashi, "Insulin-Loaded Biodegradable PLGA Microcapsules: Initial Burst Release Controlled by Hydrophilic Additives," Journal of Controlled Release, Vol. 81, No. 3, 2002, pp. 235-249.

http://dx.doi.org/10.1016/S0168-3659(02)00060-3

[8] C. Engineer, J. Parikh and A. Raval, "Effect of Copolymer Ratio on Hydrolytic Degradation of Poly (Lactideco-glycolide) from Drug Eluting Coronary Stents," Chemical Engineering Research and Design, Vol. 89, No. 3, 2011, pp. 328-334. http://dx.doi.org/10.1016/i.cherd.2010.06.013

[9] S. Xie, S. Wang, L. Zhu, F. Wang and W. Zhou, "The Effect of Glycolic Acid Monomer Ratio on the Emulsifying Activity of PLGA in Preparation of Protein-Loaded SLN," Colloids and Surfaces B: Biointerfaces, Vol. 74, No. 1, 2009, pp. 358-361.

http://dx.doi.org/10.1016/j.colsurfb.2009.08.005

[10] B. S. Zolnik and D. J. Burgess, "Evaluation of in Vivo-in Vitro Release of Dexamethasone from PLGA Microspheres," Journal of Controlled Release, Vol. 127, No. 2, 2008, pp. 137-145.

http://dx.doi.org/10.1016/j.jconrel.2008.01.004

[11] S. S. Chakravarthi and D. H. Robinson, "Enhanced Cellular Association of Paclitaxel Delivered in ChitosanPLGA Particles," International Journal of Pharmaceutics, Vol. 409, No. 1-2, 2011, pp. 111-120. http://dx.doi.org/10.1016/j.ijpharm.2011.02.034

[12] M. L. Manca, G. Loy, M. Zaru and A. M. Fadda, "Re- 
lease of Rifampicin from Chitosan, PLGA and ChitosanCoated PLGA Microparticles," Colloids and Surfaces B: Biointerfaces, Vol. 67, No. 2, 2008, pp. 166-170. http://dx.doi.org/10.1016/j.colsurfb.2008.08.010

[13] H. Nojehdehian, F. Moztarzadeh, H. Baharvand, H. Nazarian and M. Tahriri, "Preparation and Surface Characterization of Poly-1-Lysine-Coated PLGA Microsphere Scaffolds Containing Retinoic Acid for Nerve Tissue Engineering: In Vitro Study," Colloids and Surfaces B: Biointerfaces, Vol. 73, No. 1, 2009, pp. 23-29. http://dx.doi.org/10.1016/j.colsurfb.2009.04.029

[14] J. Shen and D. J. Burgess, "Accelerated in Vitro Release Testing of Implantable PLGA Microsphere/PVA Hydrogel Composite Coatings," International Journal of Pharmaceutics, Vol. 422, No. 1-2, 2012, pp. 341-348. http://dx.doi.org/10.1016/j.ijpharm.2011.10.020

[15] A. Budhian, S. J. Siegel and K. I. Winey, "Controlling the in Vitro Release Profiles for a System of HaloperidolLoaded PLGA Nanoparticles," International Journal of Pharmaceutics, Vol. 346, No. 1-2, 2008, pp. 151-159. http://dx.doi.org/10.1016/j.ijpharm.2007.06.011

[16] A. R. Ahmed, K. Elkharraz, M. Irfan and R. Bodmeier, "Reduction in Burst Release after Coating Poly(d,1-Lactide-Co-Glycolide) (PLGA) Microparticles with a DrugFree PLGA Layer," Pharmaceutical Development and
Technology, Vol. 17, No. 1, 2012, pp. 66-72. http://dx.doi.org/10.3109/10837450.2010.513989

[17] A. R. Ahmed, A. Dashevsky and R. Bodmeier, "Reduction in Burst Release of PLGA Microparticles by Incorporation into Cubic Phase-Forming Systems," European Journal of Pharmaceutics and Biopharmaceutics, Vol. 70, No. 3, 2008, pp. 765-769. http://dx.doi.org/10.1016/i.ejpb.2008.07.008

[18] R. Jeyanthi, R. C. Mehta, B. C. Thanoo and P. P. Deluca, "Effect of Processing Parameters on the Properties of Peptide-Containing PLGA Microspheres," Journal of Microencapsulation, Vol. 14, No. 2, 1997, pp. 163-174. http://dx.doi.org/10.3109/02652049709015330

[19] H. K. Makadia and S. J. Siegel, "Poly Lactic-Co-Glycolic Acid (PLGA) as Biodegradable Controlled Drug Delivery Carrier," Polymers, Vol. 3, No. 3, 2011, pp. 1377-1397. http://dx.doi.org/10.3390/polym3031377

[20] G. Mittal, D. K. Sahana, V. Bhardwaj and M. N. V. Ravi Kumar, "Estradiol Loaded PLGA Nanoparticles for Oral Administration: Effect of Polymer Molecular Weight and Copolymer Composition on Release Behavior in Vitro and in Vivo," Journal of Controlled Release, Vol. 119, No. 1, 2007, pp. 77-85.

http://dx.doi.org/10.1016/j.jconrel.2007.01.016 